\title{
Length Dependence of Charge Transport in Nanoscopic Molecular Junctions Incorporating a Series of Rigid Thiol-Terminated Norbornylogs
}

Jeremy M. Beebe ${ }^{\dagger}$, Vincent B. Engelkes ${ }^{\dagger \dagger}$, Jingquan Liu $^{\ddagger}$, J. Justin Gooding ${ }^{\ddagger}$, Paul K. Eggers $^{\ddagger}$, Yongseok Jun ${ }^{\dagger}$, Xiaoyang Zhu ${ }^{\dagger}$, Michael N. Paddon-Row ${ }^{* \ddagger}$ and C. Daniel Frisbie ${ }^{*}+$

Supporting information for the synthesis of $1,2,3,4$.
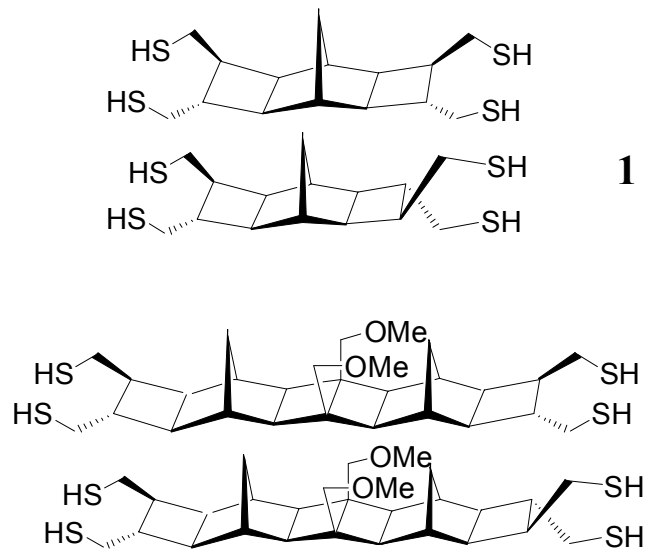

2
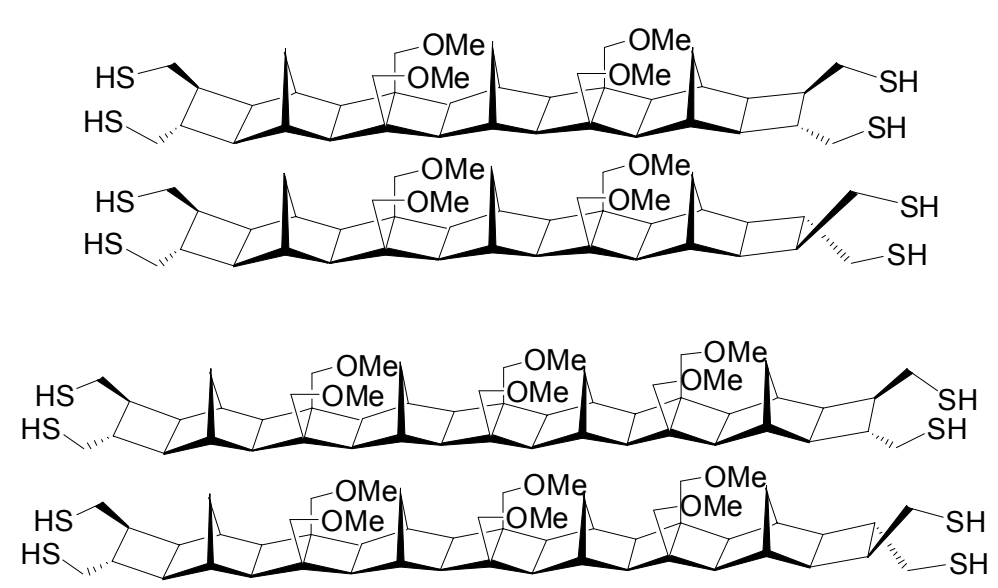

N. B. Compounds 1-4 are mixtures of two diastereomers shown above. They were not separated.

\section{Chart 1}


The synthetic strategies for product 1- $\mathbf{4}$ are depicted in Figure 1

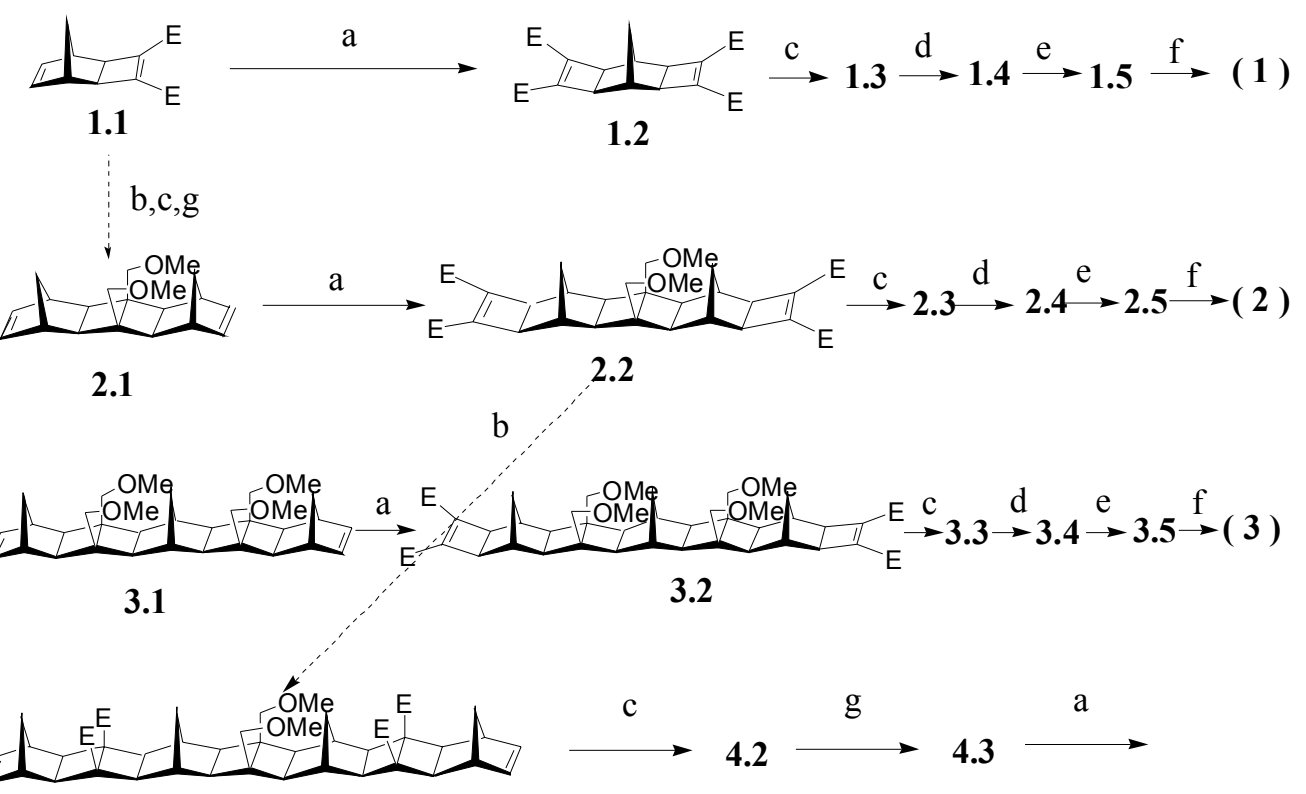

\section{1}

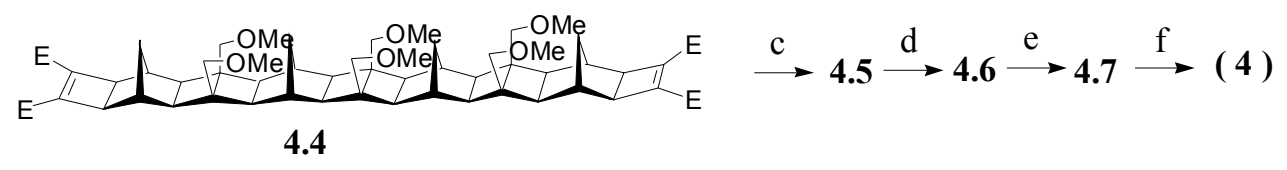

Reagents: a, DMAD, $\mathrm{RuH}_{2} \mathrm{CO}(\mathrm{PPh})_{3}$, benzene; b, quadricycline; c, $\mathrm{LiAlH}_{4}$, THF; d, TsBr, pyridine; e, $\mathrm{KSCOCH}_{3}$, DMSO; f, $\mathrm{NaOCH}_{3}, \mathrm{H}^{+}$; g, NaH, MeI, DMF.

Figure 1 


\section{Synthesis}

Compound 1.1 and 1.2 were synthesized following the procedures developed by $\mathrm{Smith}^{1}$ and Mitsudo $^{2}$. Preparations of tetraols, tetratosylates, tetrathioacetates and tetrathiols were carried out according to the procedures developed by Paddon-Row and coworkers. ${ }^{3-}$ 7

\section{The synthesis of 8-bond tetrathiol (1)}

8-bond tetraol (1.3) To a solution of $1.2(3.50 \mathrm{~g}, 9.31 \mathrm{mmol})$ in dry THF (200 mL) under argon was added $\mathrm{LiAlH}_{4}(4 \mathrm{~g}, 0.11 \mathrm{~mol})$ in small portions with care. The mixture was refluxed for $24 \mathrm{~h}$. To the cooled mixture were added $\mathrm{H}_{2} \mathrm{O}(4 \mathrm{~mL}), 15 \%$ eq. $\mathrm{NaOH}(4 \mathrm{~mL})$ and $\mathrm{H}_{2} \mathrm{O}(12 \mathrm{~mL})$ successively. The mixture was then filtered through a filter aid pad while hot. The filtrate was dried $\left(\mathrm{Na}_{2} \mathrm{SO}_{4}\right)$ and evaporated under reduced pressure to give $1.3(2.50 \mathrm{~g})$ as a thick colorless liquid, which was used for tosylation without further purification.

8-bond tetratosylate (1.4) To a cooled solution $\left(-5^{\circ} \mathrm{C}\right)$ of $\mathbf{1 . 3}(0.5 \mathrm{~g}, 1.87 \mathrm{mmol})$ in dry pyridine $(10 \mathrm{~mL})$ and $\mathrm{CH}_{2} \mathrm{Cl}_{2}(30 \mathrm{~mL}), \mathrm{TsBr}(3.52 \mathrm{~g}, 7.6 \mathrm{mmol})$ was added in small portions. The mixture was stirred under an argon atmosphere for $4 \mathrm{~h} . \mathrm{H}_{2} \mathrm{O}(50 \mathrm{~mL})$ was introduced slowly to quench the reaction, and the resulting mixture was stirred for another $1 \mathrm{~h}$. $\mathrm{CH}_{2} \mathrm{Cl}_{2}(4 \times 50 \mathrm{~mL})$ was used to extract the mixture, and the combined organic extracts was washed with $2 \mathrm{M} \mathrm{HCl}(3 \times 150 \mathrm{~mL})$, water $(100 \mathrm{~mL})$, saturated $\mathrm{NaHCO}_{3}$ aq. $(100 \mathrm{~mL})$, brine $(100 \mathrm{~mL})$ successively and then dried under $\mathrm{NaSO}_{4}$. Evaporation of the organic extracts gave a crude solid, which was chromatographed $\left(\mathrm{CH}_{2} \mathrm{Cl}_{2}\right.$ : EtOAc, 95:5) to give $1.4(0.9 \mathrm{~g}, 55 \%)$ as a white solid: ${ }^{1} \mathrm{H}$ NMR $(300 \mathrm{MHz}$, $\left.\mathrm{CDCl}_{3}\right): \delta 1.67-1.68(\mathrm{~d}, 1 \mathrm{H}), 1.70-1.72(\mathrm{~d}, 1 \mathrm{H}), 1.74-1.79(\mathrm{~m}, 2 \mathrm{H}), 1.81-1.86(\mathrm{~m}, 3 \mathrm{~h})$, 1.90-1.98 (m, 3H), 2.36-2.41 (m, 2H), 2.45 (s, 12H), 3.84-3.86 (d, 4H, $\left.\mathrm{CH}_{2}-\mathrm{S}\right), 3.92-3.99$ (m, 4H, $\left.\mathrm{CH}_{2}-\mathrm{S}\right), 7.33-7.36$ (d, 8H, aromatic), 7.72-7.76 (dd, 8H, aromatic) ${ }^{13} \mathrm{C} \mathrm{NMR}(300$ $\left.\mathrm{MHz}, \mathrm{CDCl}_{3}\right): \delta 30.37\left(\mathrm{CH}_{2}\right), 30.46\left(\mathrm{CH}_{2}\right), 34.30(\mathrm{CH}), 36.51(\mathrm{CH}), 36.58(\mathrm{CH}), 37.00$ $(\mathrm{CH}), 37.65(\mathrm{CH}), 38.24(\mathrm{CH}), 38.63(\mathrm{CH}), 39.84(\mathrm{CH}), 40.83(\mathrm{CH}), 67.85\left(\mathrm{CH}_{3} \mathrm{O}\right)$, $67.92\left(\mathrm{CH}_{3} \mathrm{O}\right), 72.27\left(\mathrm{CH}_{2} \mathrm{O}\right), 127.72(\mathrm{C}$, aromatic), $129.88(\mathrm{C}$, aromatic), $129.90(\mathrm{C}$, aromatic), $132.74\left(\mathrm{C}-\mathrm{CH}_{3}\right.$, aromatic), 144.97 (S-C, aromatic).

8-bond tetrathioacetate (1.5) To a solution of $1.4(0.59 \mathrm{~g}, 0.67 \mathrm{mmol})$ in dry DMSO (10 $\mathrm{mL})$, was added $\mathrm{KSCOCH}_{3}(0.64 \mathrm{~g}, 5.87 \mathrm{mmol})$. The mixture was heated to $70{ }^{\circ} \mathrm{C}$ and 
stirred for $24 \mathrm{~h}$ under argon. After allowing the mixture to cool to room temperature, 50 $\mathrm{mL} \mathrm{H}_{2} \mathrm{O}$ was added, and kept stirring for another $1 \mathrm{~h}$. The resulting mixture was extracted with EtOAc $(8 \times 50 \mathrm{~mL})$. The combined organic extracts was dried $\left(\mathrm{Na}_{2} \mathrm{SO}_{4}\right)$ and evaporated under reduced pressure to give $\mathbf{1 . 5}(0.34 \mathrm{~g}, 98 \%)$ as a pale brown solid: ${ }^{1} \mathrm{H}$ NMR (300 MHz, $\left.\mathrm{CDCl}_{3}\right): \delta 1.64-1.72(\mathrm{~m}, 2 \mathrm{H}), 1.80-1.89(\mathrm{~m}, 4 \mathrm{H}), 1.92-1.96(\mathrm{~m}, 3 \mathrm{H})$, 2.01-2.03 (d, $1 \mathrm{H}), 2.08(\mathrm{~s}, 1 \mathrm{H}), 2.15-2.23(\mathrm{~m}, 2 \mathrm{H}), 2.32(\mathrm{~s}, 12 \mathrm{H}), 2.76-2.87\left(\mathrm{~m}, 4 \mathrm{H}, \mathrm{CH}_{2^{-}}\right.$ S), 2.96-3.14 (m, 4H, $\left.\mathrm{CH}_{2}-\mathrm{S}\right) .{ }^{13} \mathrm{C}$ NMR $\left(300 \mathrm{MHz}, \mathrm{CDCl}_{3}\right)$ : $\delta 27.72\left(\mathrm{CH}_{2}-\mathrm{S}\right), 27.77$ $\left(\mathrm{CH}_{2}-\mathrm{S}\right), 30.52\left(\mathrm{CH}_{2}-\mathrm{S}\right), 30.57\left(\mathrm{CH}_{2}-\mathrm{S}\right), 30.73\left(\mathrm{CH}_{2}\right), 30.87\left(\mathrm{CH}_{2}\right), 34.31(\mathrm{CH}), 34.74$ $(\mathrm{CH}), 37.42(\mathrm{CH}), 38.88(\mathrm{CH}), 39.60(\mathrm{CH}), 41.07(\mathrm{CH}), 41.22(\mathrm{CH}), 41.28(\mathrm{CH}), 41.31$ $(\mathrm{CH}), 41.48(\mathrm{CH}), 43.06(\mathrm{CH}), 43.87(\mathrm{CH}), 195.55(\mathrm{C}=\mathrm{O})$.

8-bond tetrathiol (1) To a solution of $\mathbf{1 . 5}(75 \mathrm{mg}, 0.15 \mathrm{mmol})$ in dry THF (10 mL) under argon was added dry methanol $(5 \mathrm{~mL})$ and $\mathrm{NaOMe}$ solution $(1 \mathrm{~mL})$ (prepared by dissolving $0.05 \mathrm{~g} \mathrm{Na}$ in $1 \mathrm{~mL}$ dry methanol). The resulting mixture was kept stirring for $10 \mathrm{~m}$, after which $2 \mathrm{M} \mathrm{HCl}$ aqueous solution $(3 \mathrm{~mL}$ ) was added. The resulting mixture was extracted with $\mathrm{CH}_{2} \mathrm{Cl}_{2}(100 \mathrm{~mL})$, the organic part was washed with $1 \mathrm{M} \mathrm{HCl}$ solution $(2 \times 50 \mathrm{~mL})$, brine $(50 \mathrm{~mL})$ and $\mathrm{H}_{2} \mathrm{O}(50 \mathrm{~mL})$ successively. The organic phase was dried over $\mathrm{Na}_{2} \mathrm{SO}_{4}$ and evaporation gave 1 (48 mg, 96\%) as a white solid: ${ }^{1} \mathrm{H}$ NMR (300 MHz, $\left.\mathrm{CDCl}_{3}\right): \delta 1.22-1.29(\mathrm{~m}, 4 \mathrm{H}, \mathrm{SH}), 1.72-1.84(\mathrm{~m}, 6 \mathrm{H}), 1.98-2.08(\mathrm{~m}, 4 \mathrm{H}), 2.13-2.25(\mathrm{~m}$, 2H), 2.47-2.83 (m, 8H, CH $2-\mathrm{S}) .{ }^{13} \mathrm{C}$ NMR $\left(300 \mathrm{MHz}, \mathrm{CDCl}_{3}\right): \delta 23.33\left(\mathrm{CH}_{2}-\mathrm{S}\right), 23.35$ $\left(\mathrm{CH}_{2}-\mathrm{S}\right), 30.64\left(\mathrm{CH}_{2}\right), 31.10(\mathrm{CH}), 31.27(\mathrm{CH}), 33.94(\mathrm{CH}), 37.34(\mathrm{CH}), 38.53(\mathrm{CH})$, $39.30(\mathrm{CH}), 41.53(\mathrm{CH}), 42.83(\mathrm{CH}), 43.71(\mathrm{CH}), 45.31(\mathrm{CH}-\mathrm{S}), 45.52(\mathrm{CH}-\mathrm{S}), 45.56$ (CH-S), 45.69 (CH-S).

\section{The synthesis of 12-bond tetrathiol (2)}

6-bond dimethoxy diene (2.1) To a solution of 6-bond diol diene ${ }^{8}(5.45 \mathrm{~g}, 20 \mathrm{mmol})$ in DMF $(100 \mathrm{~mL})$ in ice bath was added $\mathrm{NaH}(3.3 \mathrm{~g}, 0.14 \mathrm{~mol})$ (6 g oiled, added after washing with light petroleum), after $1 \mathrm{~h}$ stirring under argon, MeI $(8 \mathrm{~mL})$ of was slowly added, kept stirring for another $5 \mathrm{~h}$, then $\mathrm{H}_{2} \mathrm{O}(100 \mathrm{~mL})$ was added to the mixture to quench the reaction, after $30 \mathrm{~m}$ stirring, the mixture was extracted with $\mathrm{CH}_{2} \mathrm{Cl}_{2}(3 \times 150$ $\mathrm{mL})$, washed with $\mathrm{H}_{2} \mathrm{O}(6 \times 200 \mathrm{~mL})$, evaporation of $\mathrm{CH}_{2} \mathrm{Cl}_{2}$ gave $2.1(5.7 \mathrm{~g}, 95 \%):{ }^{1} \mathrm{H}$ 
NMR $\left(300 \mathrm{MHz}, \mathrm{CDCl}_{3}\right): \delta 1.24-1.26(\mathrm{~d}, 2 \mathrm{H}), 1.44-1.47(\mathrm{~d}, 2 \mathrm{H}), 1.84(\mathrm{~s}, 4 \mathrm{H}), 2.87$ (4H), 3.30-3.33 (d, 10H), $6.07(4 \mathrm{H})$.

8-bond tetraester diene (2.2) To a solution of $2.1(2.1 \mathrm{~g}, 7.05 \mathrm{mmol})$ in benzene (15 $\mathrm{mL}$ ) were added DMAD (3 g, $21 \mathrm{mmol})$ and $\mathrm{RuH}_{2} \mathrm{CO}\left(\mathrm{PPh}_{3}\right)_{3}{ }^{9}(80 \mathrm{mg}, 0.09 \mathrm{mmol})$. The mixture was refluxed with stirring for 2 days, after which the solvent was removed under reduced pressure. The resulting solid was loaded onto a silica gel plug $(\mathrm{ca} .10 \mathrm{~cm})$, eluted (EtOAc: $\mathrm{CH}_{2} \mathrm{Cl}_{2}, 30: 70$ ) by $500 \mathrm{~mL}$. After evaporation of the solvent, recrystallization of the residue $\left(\mathrm{LP} / \mathrm{CH}_{2} \mathrm{Cl}_{2}\right)$ gave $2.2(3.5 \mathrm{~g}, 85 \%)$ as a white solid: ${ }^{1} \mathrm{H}$ NMR $(300 \mathrm{MHz}$, $\left.\mathrm{CDCl}_{3}\right): \delta 1.34-1.38(\mathrm{~d}, 2 \mathrm{H}), 1.51-1.55(\mathrm{~d}, 2 \mathrm{H}), 2.11(\mathrm{~s}, 4 \mathrm{H}), 2.30(\mathrm{~s}, 4 \mathrm{H}), 2.63(\mathrm{~s}, 4 \mathrm{H})$, $3.27(\mathrm{~s}, 6 \mathrm{H}), 3.32(\mathrm{~s}, 4 \mathrm{H}), 3.77(\mathrm{~s}, 12 \mathrm{H}){ }^{13} \mathrm{C} \mathrm{NMR}\left(300 \mathrm{MHz}, \mathrm{CDCl}_{3}\right)$ : $\left.\delta 26.85 \mathrm{CH}_{2}\right)$, $34.38(\mathrm{CH}), 45.91(\mathrm{C}, \mathrm{cq}), 49.05(\mathrm{CH}), 49.26(\mathrm{CH}), 51.77\left(\mathrm{OOCH}_{3}\right), 58.95\left(\mathrm{OCH}_{3}\right)$, $67.38\left(\mathrm{OCH}_{2}\right), 141.64(\mathrm{C}=\mathrm{C}), 161.46(\mathrm{COO})$.

10-bond tetraol (2.3) To a solution of $2.2(3.50 \mathrm{~g}, 6 \mathrm{mmol})$ in dry THF $(150 \mathrm{~mL})$ under argon was added $\mathrm{LiAlH}_{4}(3 \mathrm{~g}, 80 \mathrm{mmol})$ in small portions. The mixture was refluxed for $24 \mathrm{~h}$, and then allowed to cool to room temperature. To the cooled mixture were added $\mathrm{H}_{2} \mathrm{O}(3 \mathrm{~mL}), 15 \%$ aq. $\mathrm{NaOH}(3 \mathrm{~mL})$ and $\mathrm{H}_{2} \mathrm{O}(9 \mathrm{~mL})$ was added successively with care. The mixture was then filtered, dried $\left(\mathrm{Na}_{2} \mathrm{SO}_{4}\right)$, and evaporated under reduced pressure to give 2.3 (2.50 g, 100\%), which was used for tosylation without purification.

12-bond tetratosylate (2.4) To a cooled $\left(-5^{\circ} \mathrm{C}\right)$ solution of $2.3(2.5 \mathrm{~g}, 5.3 \mathrm{mmol})$ in dry pyridine $(10 \mathrm{~mL})$ and $\mathrm{CH}_{2} \mathrm{Cl}_{2}(100 \mathrm{~mL}), \mathrm{TsBr}(6 \mathrm{~g}, 26 \mathrm{mmol})$ was added in small portions, the mixture was stirred under an argon atmosphere for $4 \mathrm{~h}, \mathrm{H}_{2} \mathrm{O}(100 \mathrm{~mL})$ was slowly introduced, the resulting mixture was kept stirring for another $1 \mathrm{~h} . \mathrm{CH}_{2} \mathrm{Cl}_{2}(4 \times$ $100 \mathrm{~mL}$ ) was used to extract the mixture, and the combined organic extracts was washed with $2 \mathrm{M} \mathrm{HCl}(3 \times 150 \mathrm{~mL})$, water $(100 \mathrm{~mL})$, saturated $\mathrm{NaHCO}_{3}$ aq. $(100 \mathrm{~mL})$, brine $(100 \mathrm{~mL})$ successively and then dried over $\mathrm{NaSO}_{4}$. Evaporation of the combined organic extracts gave crude product, which was chromatographed $\left(\mathrm{CH}_{2} \mathrm{Cl}_{2}\right.$ : EtOAc, $\left.96: 4\right)$ to give pure $2.4(1.5 \mathrm{~g}, 27 \%)$ as a white solid: ${ }^{1} \mathrm{H}$ NMR $\left(300 \mathrm{MHz}, \mathrm{CDCl}_{3}\right): \delta 1.62-1.69(\mathrm{~m}, 4 \mathrm{H})$, 1.79-1.83 (m, 6H), 1.91-2.02 (m, 5H), 2.05-2.10 (d, 2H), 2.35-2.38 (m, 2H), $2.43(\mathrm{~s}, 12 \mathrm{H}$, $\mathrm{CH}_{3}-\mathrm{CO}$ ), 3.82-3.84 (d, $\left.4 \mathrm{H}, \mathrm{CH}_{2}-\mathrm{S}\right), 3.98-4.08\left(4 \mathrm{H}, \mathrm{CH}_{2}-\mathrm{S}\right), 7.31-7.34(\mathrm{~d}, 8 \mathrm{H}, \mathrm{CH}=\mathrm{CH})$, 7.72-7.74 (d, 8H, $\mathrm{CH}=\mathrm{CH}){ }^{13} \mathrm{C} \mathrm{NMR}\left(300 \mathrm{MHz}, \mathrm{CDCl}_{3}\right): \delta 30.81\left(\mathrm{CH}_{2}\right), 35.41(\mathrm{CH})$, $35.75(\mathrm{CH}), 36.96(\mathrm{CH}), 38.09(\mathrm{CH}), 38.43(\mathrm{CH}), 38.48(\mathrm{CH}), 40.43(\mathrm{C}, \mathrm{cq}), 40.69(\mathrm{C}$, 
cq), $47.93(\mathrm{CH}), 48.16(\mathrm{CH}), 48.40(\mathrm{CH}), 48.48(\mathrm{CH}), 48.54(\mathrm{CH}), 49.04(\mathrm{CH}), 49.18$ $(\mathrm{CH}), 49.27(\mathrm{CH}), 53.41\left(\mathrm{OCH}_{3}\right), 58.89\left(\mathrm{OCH}_{3}\right), 66.53\left(\mathrm{OCH}_{2}\right), 67.37\left(\mathrm{OCH}_{2}\right), 68.19(\mathrm{O}-$ $\left.\mathrm{SO}_{2}-\mathrm{CH}_{2}\right), 72.59\left(\mathrm{O}-\mathrm{SO}_{2}-\mathrm{CH}_{2}\right), 127.69(\mathrm{CH}=\mathrm{CH}), 127.79(\mathrm{CH}=\mathrm{CH}), 129.84(\mathrm{CH}=\mathrm{CH})$, $132.67(\mathrm{CH}=\mathrm{CH}), 132.88\left(\mathrm{C}-\mathrm{CH}_{3}\right), 144.81(\mathrm{CO}-\mathrm{S})$

12-bond tetrathioacetate (2.5) To a solution of $2.4(1 \mathrm{~g}, 0.92 \mathrm{mmol})$ in dry DMSO (10 $\mathrm{mL}$ ) was added $\mathrm{KSCOCH}_{3}(0.84 \mathrm{~g}, 7.4 \mathrm{mmol})$. The mixture was heated to $70{ }^{\circ} \mathrm{C}$ and stirred for $24 \mathrm{~h}$ under argon. After cooling down to room temperature. $\mathrm{H}_{2} \mathrm{O}(50 \mathrm{~mL})$ was added, kept stirring for another $1 \mathrm{~h}$. The resulting mixture was extracted with EtOAc $(8 \times$ $50 \mathrm{~mL})$. The organic extracts was dried $\left(\mathrm{Na}_{2} \mathrm{SO}_{4}\right)$ and evaporated under reduced pressure to give pure $2.5(0.63 \mathrm{~g}, 97 \%)$ as a pale brown solid: ${ }^{1} \mathrm{H} \mathrm{NMR}\left(300 \mathrm{MHz}, \mathrm{CDCl}_{3}\right)$ : $\delta$ 1.65-1.67 (d, 1H), 1.71-1.73 (d, 1H), 1.75-1.77 (d, 2H), 1.80-1.85 (m, 3H), 1.89-1.91 (t, $3 \mathrm{H}), 2.01-2.05(\mathrm{~m}, 4 \mathrm{H}), 2.20-2.25(\mathrm{~m}, 2 \mathrm{H}), 2.28-2.31(\mathrm{~m}, 12 \mathrm{H}), 2.80-2.87\left(\mathrm{~m}, 4 \mathrm{H}, \mathrm{CH}_{2}-\right.$ S), 2.94-3.01 (dd, $2 \mathrm{H}, \mathrm{CH}_{2}-\mathrm{S}$ ), 3.10-3.18 (dd, $\left.2 \mathrm{H}, \mathrm{CH}_{2}-\mathrm{S}\right), 3.25-3.27$ (m, $\left.6 \mathrm{H}\right), 3.29-3.34$ (m, 4H). ${ }^{13} \mathrm{C}$ NMR $\left(300 \mathrm{MHz}, \mathrm{CDCl}_{3}\right): \delta 27.67\left(\mathrm{COCH}_{3}\right), 30.48\left(\mathrm{CH}_{2}\right), 30.57\left(\mathrm{CH}_{2}\right)$, $30.62\left(\mathrm{CH}_{2}\right), 31.12\left(\mathrm{CH}_{2}\right), 34.85(\mathrm{CH}), 35.44(\mathrm{CH}), 38.77(\mathrm{CH}), 39.33(\mathrm{CH}), 41.10(\mathrm{CH})$, $41.67(\mathrm{CH}), 43.52(\mathrm{C}, \mathrm{cq}), 48.31(\mathrm{CH}), 48.41(\mathrm{CH}), 48.51(\mathrm{CH}), 49.13(\mathrm{CH}), 49.34(\mathrm{CH})$, $58.86\left(\mathrm{OCH}_{3}\right), 58.89\left(\mathrm{OCH}_{3}\right), 67.54\left(\mathrm{OCH}_{2}\right), 195.72(\mathrm{C}=\mathrm{O}), 195.96(\mathrm{C}=\mathrm{O})$.

12-bond tetrathiol (2) To a solution of 2.5 (400 mg, $0.57 \mathrm{mmol})$ in dry THF (12 mL) under argon was added dry methanol $(5 \mathrm{~mL})$ and $\mathrm{NaOMe}$ solution $(1 \mathrm{~mL})$ (prepared by dissolving $0.05 \mathrm{~g} \mathrm{Na}$ in $1 \mathrm{~mL}$ dry methanol). The resulting mixture was kept stirring for $10 \mathrm{~m}$, after which $2 \mathrm{M} \mathrm{HCl}$ aqueous solution $(20 \mathrm{~mL})$ was added. The resulting mixture was extracted with $\mathrm{CH}_{2} \mathrm{Cl}_{2}(3 \times 50 \mathrm{~mL})$, the organic part was washed with argondegassed $1 \mathrm{M} \mathrm{HCl}$ solution $(2 \times 50 \mathrm{~mL})$, brine $(50 \mathrm{~mL})$ and $\mathrm{H}_{2} \mathrm{O}(50 \mathrm{~mL})$ successively. The organic phase was dried over $\mathrm{Na}_{2} \mathrm{SO}_{4}$, and evaporated to give $2(267 \mathrm{mg}, 88 \%)$ as a white solid: ${ }^{1} \mathrm{H}$ NMR $\left(300 \mathrm{MHz}, \mathrm{CDCl}_{3}\right.$ ): $\delta 1.22-1.32$ (m, 4H, SH), $1.74(\mathrm{~S}, 4 \mathrm{H}), 1.80$ $1.86(\mathrm{~m}, 3 \mathrm{H}), 1.94-1.97(\mathrm{~m}, 2 \mathrm{H}), 2.0 \mathrm{O}-2.03(\mathrm{~m}, 2 \mathrm{H}), 2.04-2.08(\mathrm{~m}, 4 \mathrm{H}), 2.17-2.20(\mathrm{t}$, $3 \mathrm{H}), 2.23-2.32(\mathrm{~m}, 2 \mathrm{H}), 2.49-2.72\left(\mathrm{~m}, 7 \mathrm{H}, \mathrm{CH}_{2}-\mathrm{S}\right), 2.82-2.87$ (m, $\left.1 \mathrm{H}, \mathrm{CH}_{2}-\mathrm{S}\right) .{ }^{13} \mathrm{C} \mathrm{NMR}$ $\left(300 \mathrm{MHz}, \mathrm{CDCl}_{3}\right): \delta 23.40\left(\mathrm{CH}_{2}-\mathrm{S}\right), 30.71\left(\mathrm{CH}_{2}\right), 31.21\left(\mathrm{CH}_{2}\right), 35.30(\mathrm{CH}), 38.94$ $(\mathrm{CH}), 39.00(\mathrm{CH}), 43.33(\mathrm{CH}), 45.53(\mathrm{CH}), 45.96(\mathrm{CH}), 48.43(\mathrm{CH}), 48.50(\mathrm{CH}), 48.58$ $(\mathrm{CH}), 48.74(\mathrm{CH}), 49.38(\mathrm{C}, \mathrm{cq}), 49.62(\mathrm{C}, \mathrm{cq}), 58.88\left(\mathrm{OCH}_{3}\right), 67.68\left(\mathrm{OCH}_{2}\right)$. 


\section{The synthesis of 16-bond tetrathiol (3)}

10-bond tetramethoxy diene (3.1) To a solution of 10-bond tetraol diene ${ }^{8}$ (3 g, 6.7 mmol) in DMF (150 mL) in ice bath was added $\mathrm{NaH}$ (3.3 g, $0.14 \mathrm{~mol})$ (6 g oiled, added after washing with light petroleum), after $1 \mathrm{~h}$ stirring under argon, MeI (8 mL) of was slowly added, kept stirring for another $5 \mathrm{~h}$, then $\mathrm{H}_{2} \mathrm{O}(100 \mathrm{~mL})$ was added to the mixture to quench the reaction, after $30 \mathrm{~m}$ stirring, the mixture was extracted with $\mathrm{CH}_{2} \mathrm{Cl}_{2}(3 \times$ $150 \mathrm{~mL})$, washed with $\mathrm{H}_{2} \mathrm{O}(6 \times 200 \mathrm{~mL})$, evaporation of $\mathrm{CH}_{2} \mathrm{Cl}_{2}$ gave $3.1(3.10 \mathrm{~g}, 91 \%)$ : ${ }^{1} \mathrm{H}$ NMR (300 MHz, $\left.\mathrm{CDCl}_{3}\right): \delta 1.16-1.87(\mathrm{~d}, 2 \mathrm{H}), 1.43-1.47$ (d, 2H), $1.79(\mathrm{~s}, 2 \mathrm{H}), 1.85$ $(4 \mathrm{H}), 2.00(4 \mathrm{H}), 2.33(4 \mathrm{H}), 2.85(4 \mathrm{H}), 3.30-4.42(20 \mathrm{H}), 6.04(4 \mathrm{H}) .{ }^{13} \mathrm{C}$ NMR $(300 \mathrm{MHz}$, $\left.\mathrm{CDCl}_{3}\right): \delta 31.01\left(\mathrm{CH}_{2}\right), 37.16(\mathrm{CH}), 41.87(\mathrm{CH}), 42.34(\mathrm{CH}), 45.44(\mathrm{CH}), 46.30(\mathrm{CH})$, $49.23(\mathrm{C}, \mathrm{cq}), 58.71\left(\mathrm{CH}_{3}\right), 67.58\left(\mathrm{CH}_{2} \mathrm{O}, 136.35(\mathrm{CH}=\mathrm{CH})\right.$

12-bond tetraester (3.2) To a solution of 3.1 (2 g, $3.97 \mathrm{mmol})$ in benzene (30 mL) were added DMAD (1.35 g, $9.52 \mathrm{mmol})$ and $\mathrm{RuH}_{2} \mathrm{CO}\left(\mathrm{PPh}_{3}\right)_{3}(80 \mathrm{mg}, 0.09 \mathrm{mmol})$ as catalyst. The mixture was refluxed with stirring for 2 days, after which the solvent was removed under reduced pressure. The resulting solid was loaded onto a silica gel plug $(\mathrm{ca} .10 \mathrm{~cm})$, eluted (EtOAc: $\mathrm{CH}_{2} \mathrm{Cl}_{2}, 30: 70$ ) by $800 \mathrm{~mL}$. After evaporation of the solvent, recrystallization of the residue $\left(\mathrm{LP} / \mathrm{CH}_{2} \mathrm{Cl}_{2}\right)$ gave $\mathbf{3 . 2}(2.88 \mathrm{~g}, 92 \%)$ as a white solid: ${ }^{1} \mathrm{H}$ NMR (300 MHz, $\left.\mathrm{CDCl}_{3}\right): \delta 1.31-1.35(\mathrm{~d}, 2 \mathrm{H}), 1.58-1.62(\mathrm{~d}, 2 \mathrm{H}), 1.75-.76(\mathrm{~d}, 2 \mathrm{H}), 2.09$ $(\mathrm{s}, 8 \mathrm{H}), 2.24(\mathrm{~s}, 2 \mathrm{H}), 2.30(\mathrm{~s}, 4 \mathrm{H}), 2.62(\mathrm{~s}, 4 \mathrm{H}), 3.27-3.29\left(\mathrm{~d}, 12 \mathrm{H}, 4 \times \mathrm{OCH}_{3}\right), 3.36(\mathrm{~s}$, $\left.8 \mathrm{H}, 4 \times \mathrm{OCH}_{2}\right), 3.78\left(\mathrm{~s}, 12 \mathrm{H}, 4 \times \mathrm{OOCH}_{3}\right) \cdot{ }^{13} \mathrm{C} \mathrm{NMR}\left(300 \mathrm{MHz}, \mathrm{CDCl}_{3}\right): \delta 34.45\left(\mathrm{CH}_{2}\right)$, $46.01(\mathrm{CH}), 48.97(\mathrm{CH}), 49.17(\mathrm{CH}), 49.51(\mathrm{C}, \mathrm{cq}), 51.66\left(\mathrm{CH}_{3}\right), 58.75\left(\mathrm{OCH}_{3}\right), 67.64$ $\left(\mathrm{OCH}_{2}\right), 141.65(\mathrm{C}=\mathrm{C}), 161.49(\mathrm{C}=\mathrm{O})$.

16bond tetraol (3.3) To a solution of $3.2(0.6 \mathrm{~g}, 0.76 \mathrm{mmol})$ in dry THF $(100 \mathrm{~mL})$ under argon was added $\mathrm{LiAlH}_{4}(1 \mathrm{~g}, 0.026 \mathrm{~mol})$ in small portions. The mixture was refluxed for $24 \mathrm{~h}$, and then allowed to cool to room temperature. To the cooled mixture were added $\mathrm{H}_{2} \mathrm{O}(1 \mathrm{~mL}), 15 \%$ eq. $\mathrm{NaOH}(1 \mathrm{~mL})$ and $\mathrm{H}_{2} \mathrm{O}(3 \mathrm{~mL})$ successively with care. The mixture was then filtered through a filter aid pad while hot, dried $\left(\mathrm{Na}_{2} \mathrm{SO}_{4}\right)$ and evaporated under reduced pressure to give $\mathbf{3 . 3}(0.52 \mathrm{~g}, 100 \%)$, which was used for tosylation without further purification.

16-bond tetratosylate (3.4) To a cooled $\left(-5^{\circ} \mathrm{C}\right)$ solution of $3.3(0.51 \mathrm{~g}, 1.87 \mathrm{mmol})$ in dry pyridine $(10 \mathrm{~mL})$ and $\mathrm{CH}_{2} \mathrm{Cl}_{2}(40 \mathrm{~mL})$, TsBr $(2 \mathrm{~g}, 8.5 \mathrm{mmol})$ was added in small 
portions. The mixture was stirred under an argon atmosphere for $4 \mathrm{~h} . \mathrm{H}_{2} \mathrm{O}(50 \mathrm{~mL})$ was slowly introduced, and the resulting mixture was kept stirring for another $1 \mathrm{~h} . \mathrm{CH}_{2} \mathrm{Cl}_{2}(4$ $\times 50 \mathrm{~mL}$ ) was used to extract the mixture, and the combined organic extracts was washed with $2 \mathrm{M} \mathrm{HCl}(3 \times 100 \mathrm{~mL})$, water $(100 \mathrm{~mL})$, saturated $\mathrm{NaHCO}_{3}$ aq. $(100 \mathrm{~mL})$, brine $(100 \mathrm{~mL})$ successively and then dried under $\mathrm{Na}_{2} \mathrm{SO}_{4}$. Evaporation of the organic extracts gave a crude solid, which chromatographed $\left(\mathrm{CH}_{2} \mathrm{Cl}_{2}\right.$ : EtOAc, $\left.90: 10\right)$ to give $3.4(0.32 \mathrm{~g}$, 34\%) as a white solid: ${ }^{1} \mathrm{H}$ NMR (300 MHz, $\mathrm{CDCl}_{3}$ ): $\delta$ 1.58-1.59 (d, 2H), 1.64-1.69 (d, $4 \mathrm{H}), 1.76-1.88$ (m, 8H), 1.98-2.03 (m, 10H), 2.09-2.13 (d, 4H), 2.22-2.25 (m, 1H), 2.36$2.41(\mathrm{~m}, 1 \mathrm{H}), 2.44(\mathrm{~s}, 12 \mathrm{H}), 3.23-3.24\left(\mathrm{~d}, 12 \mathrm{H}, \mathrm{OCH}_{3}\right), 3.27-3.29\left(\mathrm{~d}, 8 \mathrm{H}, \mathrm{OCH}_{2}\right), 3.84-$ 3.86 (d, 4H, CH2-S), 4.03-4.11 (m, 4H, CH2-S), 7.32-7.34 (dd, 8H, $\mathrm{CH}=\mathrm{CH}$ ), 7.72-7.75 $(\mathrm{dd}, 8 \mathrm{H}, \mathrm{CH}=\mathrm{CH}) .{ }^{13} \mathrm{C}$ NMR $\left(300 \mathrm{MHz}, \mathrm{CDCl}_{3}\right): \delta 21.54\left(\mathrm{CH}_{3}\right), 30.72(\mathrm{CH}), 35.52$ $(\mathrm{CH}), 36.67(\mathrm{CH}), 37.06(\mathrm{CH}), 38.21(\mathrm{CH}), 38.53(\mathrm{CH}), 38.61(\mathrm{CH}), 40.82(\mathrm{CH}), 48.28$ $(\mathrm{CH}), 48.62(\mathrm{C}, \mathrm{cq}), 48.69(\mathrm{C}, \mathrm{cq}), 49.04(\mathrm{CH}), 49.28(\mathrm{CH}), 49.39(\mathrm{CH}), 58.72(\mathrm{CH})$, $67.62\left(\mathrm{OCH}_{3}\right), 68.23\left(\mathrm{OCH}_{3}\right), 72.65\left(\mathrm{COCH}_{3}\right), 127.70\left(\mathrm{C}-\mathrm{CH}_{3}\right), 127.80\left(\mathrm{C}-\mathrm{CH}_{3}\right), 129.80$ $(\mathrm{CH}=\mathrm{CH}), 132.84(\mathrm{CH}=\mathrm{CH}), 133.05(\mathrm{~S}-\mathrm{C}), 144.72(\mathrm{~S}-\mathrm{C})$.

16-bond tetrathioacetate (3.5) To a solution of $3.4(0.32 \mathrm{~g}, 0.25 \mathrm{mmol})$ in dry DMSO $(10 \mathrm{~mL})$, was added $\mathrm{KSCOCH}_{3}(0.3 \mathrm{~g}, 2.6 \mathrm{mmol})$. The mixture was heated to $70{ }^{\circ} \mathrm{C}$ and stirred for $24 \mathrm{~h}$ under argon. After allowing the mixture to cool to room temperature, $\mathrm{H}_{2} \mathrm{O}$ $(50 \mathrm{~mL})$ was added, kept stirring for another $1 \mathrm{~h}$. The resulting mixture was extracted with EtOAc $(8 \times 30 \mathrm{~mL})$. The organic extracts was dried over $\mathrm{Na}_{2} \mathrm{SO}_{4}$ and evaporated under reduced pressure to give $3.5(0.20 \mathrm{~g}, 88 \%)$ as a pale brown solid: ${ }^{1} \mathrm{H}$ NMR (300 $\left.\mathrm{MHz}, \mathrm{CDCl}_{3}\right): \delta 1.70-1.71(\mathrm{~m}, 2 \mathrm{H}), 1.75-1.79(\mathrm{~m}, 4 \mathrm{H}), 1.81-1.88(\mathrm{t}, 4 \mathrm{H}), 1.91-1.95(\mathrm{t}$, $2 \mathrm{H}), 1.97-1.99(\mathrm{~d}, 2 \mathrm{H}), 2.01-2.06(\mathrm{~m}, 9 \mathrm{H}), 2.13-2.16(\mathrm{~d}, 4 \mathrm{H}), 2.21-2.26(\mathrm{t}, 2 \mathrm{H}), 2.28-2.31$ (d, 12H), 2.84-2.88 (m, 4H), 2.96-3.02 (dd, $2 \mathrm{H}), 3.11-3.18(\mathrm{dd}, 2 \mathrm{H}), 3.25-3.26(\mathrm{~d}, 12 \mathrm{H}$, $\left.\mathrm{OCH}_{3}\right), 3.30-3.40\left(\mathrm{~m}, 8 \mathrm{H}, \mathrm{OCH}_{2}\right) .{ }^{13} \mathrm{C}$ NMR $\left(300 \mathrm{MHz}, \mathrm{CDCl}_{3}\right)$ : $\delta 27.71\left(\mathrm{CH}_{3} \mathrm{CO}\right)$, $30.43\left(\mathrm{CH}_{2}\right), 30.51\left(\mathrm{CH}_{2}\right), 30.57\left(\mathrm{CH}_{2}\right), 30.92\left(\mathrm{CH}_{2}\right), 31.10\left(\mathrm{CH}_{2}\right), 34.88(\mathrm{CH}), 35.53$ $(\mathrm{CH}), 36.68(\mathrm{CH}), 38.88(\mathrm{CH}), 39.37(\mathrm{CH}), 41.16(\mathrm{CH}), 41.70(\mathrm{CH}), 43.62(\mathrm{CH}), 48.54$ (C, cq), 48.59 (C, cq), 48.64 (C, cq), 48.68 (C, cq), $49.11\left(\mathrm{SCH}_{2}\right), 49.20\left(\mathrm{SCH}_{2}\right), 49.32$ $\left(\mathrm{SCH}_{2}\right), 49.48\left(\mathrm{SCH}_{2}\right), 58.69\left(\mathrm{OCH}_{3}\right), 67.72\left(\mathrm{OCH}_{2}\right), 195.65(\mathrm{C}=\mathrm{O}), 195.86(\mathrm{C}=\mathrm{O})$.

16-bond tetrathiol (3) To a solution of 3.5 (74 $\mathrm{mg}, 0.08 \mathrm{mmol})$ in dry THF (5 mL) under argon was added dry methanol $(5 \mathrm{~mL})$ and $\mathrm{NaOMe}$ solution $(1 \mathrm{~mL})$ (prepared by 
dissolving $0.05 \mathrm{~g} \mathrm{Na}$ in $1 \mathrm{~mL}$ dry methanol). The resulting mixture was kept stirring for $10 \mathrm{~m}$, after which $1 \mathrm{M} \mathrm{HCl}$ aqueous solution $(10 \mathrm{~mL})$ was added. The whole mixture was extracted with $\mathrm{CH}_{2} \mathrm{Cl}_{2}(100 \mathrm{~mL}$ ), the organic phase was washed with $1 \mathrm{M} \mathrm{HCl}$ solution (2 $\times 50 \mathrm{~mL})$, brine $(50 \mathrm{~mL})$ and $\mathrm{H}_{2} \mathrm{O}(50 \mathrm{~mL})$ successively. The organic phase was dried over $\mathrm{Na}_{2} \mathrm{SO}_{4}$ and evaporated to give $3(60 \mathrm{mg}, 97 \%)$ as a white solid: ${ }^{1} \mathrm{H}$ NMR (300 $\left.\mathrm{MHz}, \mathrm{CDCl}_{3}\right): \delta 1.22-1.31(\mathrm{~m}, 4 \mathrm{H}, \mathrm{SH}), 1.73-1.77(\mathrm{~d}, 6 \mathrm{H}), 1.79-1.86(\mathrm{~m}, 3 \mathrm{H}), 1.95-1.97$ $(\mathrm{t}, 4 \mathrm{H}), 2.01-2.04(\mathrm{~d}, 6 \mathrm{H}), 2.08(\mathrm{~d}, 3 \mathrm{H}), 2.16-2.33(\mathrm{~m}, 6 \mathrm{H}, \mathrm{CH}), 2.53-2.67\left(\mathrm{~m}, 6 \mathrm{H}, \mathrm{CH}_{2^{-}}\right.$ S), 2.80-2.87 (m, $\left.2 \mathrm{H}, \mathrm{CH}_{2}-\mathrm{S}\right), 3.25-3.26\left(\mathrm{~d}, 12 \mathrm{H}, \mathrm{OCH}_{3}\right), 3.31-3.40\left(\mathrm{~m}, 8 \mathrm{H}, \mathrm{OCH}_{2}\right) .{ }^{13} \mathrm{C}$ NMR (300 MHz, $\left.\mathrm{CDCl}_{3}\right): 23.40\left(\mathrm{CH}_{2}-\mathrm{S}\right), 30.72\left(\mathrm{CH}_{2}\right), 31.76\left(\mathrm{CH}_{2}\right), 35.56(\mathrm{CH}), 36.71$ $(\mathrm{CH}), 39.01(\mathrm{CH}), 43.37(\mathrm{CH}), 45.55(\mathrm{CH}), 46.01(\mathrm{CH}), 48.61(\mathrm{CH}), 48.68(\mathrm{CH}), 49.13$ (C, cq), $49.38(\mathrm{C}, \mathrm{cq}), 49.63(\mathrm{C}, \mathrm{cq}), 58.72\left(\mathrm{OCH}_{3}\right), 67.78\left(\mathrm{OCH}_{2}\right), 67.85\left(\mathrm{OCH}_{2}\right)$.

\section{The synthesis of 20-bond tetrathiol (4)}

14-bond dimethoxy-tetraester diene (4.1) To deoxygenated quadricycline (20 g, 0.22 mol) was added $2.2(7.5 \mathrm{~g}, 12.7 \mathrm{mmol})$. The mixture was refluxed under argon atmosphere for 7 days, after which the unreacted quadricycline was removed under reduced pressure. The resulting solid compound was recrystalized (ethanol/ $/ \mathrm{CH}_{2} \mathrm{Cl}_{2}$ ) to give $4.1(8.5 \mathrm{~g}, 86 \%)$ as a white solid: ${ }^{1} \mathrm{H}$ NMR $\left(300 \mathrm{MHz}, \mathrm{CDCl}_{3}\right): \delta 1.05-1.08(\mathrm{~d}, 2 \mathrm{H})$, 1.59-1.63 (d, 2H), 1.74-1.75 (d, 2H), $2.03(\mathrm{~s}, 4 \mathrm{H}), 2.10$ (s, 4H), 2.17 (s, 4H), 2.25-2.26 (d, $6 \mathrm{H}), 2.79(\mathrm{~s}, 4 \mathrm{H}), 3.2\left(\mathrm{~s}, 6 \mathrm{H}, \mathrm{OCH}_{3}\right), 3.25\left(\mathrm{~s}, 4 \mathrm{H}, \mathrm{OCH}_{2}\right), 3.70\left(\mathrm{~s}, 12 \mathrm{H}, \mathrm{OOCH}_{3}\right), 6.04(\mathrm{~s}$, $4 \mathrm{H}, \mathrm{CH}=\mathrm{CH}) .{ }^{13} \mathrm{C}$ NMR $\left(300 \mathrm{MHz}, \mathrm{CDCl}_{3}\right): \delta 29.35\left(\mathrm{CH}_{2}\right), 38.26(\mathrm{CH}), 40.53(\mathrm{CH})$, $43.78(\mathrm{CH}), 47.08(\mathrm{C}, \mathrm{cq}), 48.38(\mathrm{CH}), 48.63(\mathrm{CH}), 50.45(\mathrm{CH}), 50.70(\mathrm{CH}), 53.68$ $\left(\mathrm{OOCH}_{3}\right), 58.73\left(\mathrm{OCH}_{2}\right), 67.08\left(\mathrm{OCH}_{2}\right), 136.02(\mathrm{C}=\mathrm{C}), 170.61(\mathrm{COO})$.

14-bond dimethoxy tetraol (4.2) To a solution of $4.1(4 \mathrm{~g}, 5.2 \mathrm{mmol})$ in dry THF $(100 \mathrm{~mL})$ under argon was added $\mathrm{LiAlH}_{4}(3 \mathrm{~g}, 79 \mathrm{mmol})$ in small portions. The mixture was refluxed for $20 \mathrm{~h}$. To the cooled mixture were added $\mathrm{H}_{2} \mathrm{O}(3 \mathrm{~mL}), 15 \%$ eq. $\mathrm{NaOH}$ ( 3 $\mathrm{mL})$ and $\mathrm{H}_{2} \mathrm{O}(9 \mathrm{~mL})$ successively with care. The mixture was then filtered through a filter aid pad while hot, the filtrate was dried $\left(\mathrm{Na}_{2} \mathrm{SO}_{4}\right)$ and evaporated under reduced pressure to give 4.2 (3.42 g, 100\%), which was used for methoxylation in the next step without purification. 
14-bond hexamethoxy diene (4.3) To a cooled $\left(-5^{\circ} \mathrm{C}\right)$ solution of $4.2(2.55 \mathrm{~g}, 3.9 \mathrm{mmol})$ in dry DMF $(150 \mathrm{~mL})$ was slowly added $\mathrm{NaH}(2.2 \mathrm{~g}, 92 \mathrm{mmol})(4 \mathrm{~g}$ oiled $\mathrm{NaH}$, added after washing with light petroleum). The mixture was stirred at $0{ }^{\circ} \mathrm{C}$ for $1 \mathrm{~h}$, after which MeI $(5 \mathrm{~mL})$ was added in small portions with care. The resulting mixture was stirred for another $20 \mathrm{~h}$, and then slowly added $\mathrm{H}_{2} \mathrm{O}(100 \mathrm{~mL})$ to quench the reaction. $\mathrm{CH}_{2} \mathrm{Cl}_{2}(4 \times$ $100 \mathrm{~mL}$ ) was used to extract the mixture. The combined organic extracts was washed with $\mathrm{H}_{2} \mathrm{O}(6 \times 200 \mathrm{~mL})$, dried under $\mathrm{Na}_{2} \mathrm{SO}_{4}$ and evaporated to give crude product, which was recrystallised (ethanol/ $\mathrm{CH}_{2} \mathrm{Cl}_{2}$ ) to give pure $4.3(2 \mathrm{~g}, 72 \%)$ as a white solid: ${ }^{1} \mathrm{H} \mathrm{NMR}$ $\left(300 \mathrm{MHz}, \mathrm{CDCl}_{3}\right): \delta 1.15-1.18(\mathrm{~d}, 2 \mathrm{H}), 1.43-1.46(\mathrm{~d}, 2 \mathrm{H}), 1.72-1.76(\mathrm{~d}, 2 \mathrm{H}), 1.82-1.85$ (d, 6H), 2.00 (s, 4H), $2.06(\mathrm{~s}, 4 \mathrm{H}), 2.28(\mathrm{~s}, 4 \mathrm{H}), 3.28-3.29$ (m, 18H, $\left.\mathrm{OCH}_{3}\right), 3.37-3.41(\mathrm{~m}$, $\left.12 \mathrm{H}, \mathrm{OCH}_{2}\right), 6.04(\mathrm{~s}, \mathrm{CH}=\mathrm{CH}) .{ }^{13} \mathrm{C}$ NMR $\left(300 \mathrm{MHz}, \mathrm{CDCl}_{3}\right): \delta 30.90\left(\mathrm{CH}_{2}\right), 37.03$ $(\mathrm{CH}), 41.86(\mathrm{CH}), 42.34(\mathrm{CH}), 45.42(\mathrm{CH}), 46.29(\mathrm{C}, \mathrm{cq}), 48.82(\mathrm{CH}), 49.16(\mathrm{CH}), 49.53$ $(\mathrm{CH}), 58.59\left(\mathrm{OCH}_{2}\right), 58.70\left(\mathrm{OCH}_{2}\right), 67.57\left(\mathrm{OCH}_{2}\right), 67.92\left(\mathrm{OCH}_{2}\right), 136.35(\mathrm{CH}=\mathrm{CH})$.

16-bond hexamethoxy-tetraester diene (4.4) To a solution of $4.3(0.92 \mathrm{~g}, 1.3 \mathrm{mmol})$ in benzene $(20 \mathrm{~mL})$ were added DMAD $(0.5 \mathrm{~g}, 3.5 \mathrm{mmol})$ and $\mathrm{RuH}_{2} \mathrm{CO}\left(\mathrm{PPh}_{3}\right)_{3}(25 \mathrm{mg}$, $0.03 \mathrm{mmol}$ ). The mixture was refluxed with stirring for 2 days, after which the solvent was removed under reduced pressure. The resulting solid was loaded onto a silica gel plug (ca $10 \mathrm{~cm}$ ), eluted (EtOAc: $\mathrm{CH}_{2} \mathrm{Cl}_{2}, 30: 70$ ) by $200 \mathrm{~mL}$. After evaporation of the solvent, recrystallization $\left(\mathrm{LP} / \mathrm{CH}_{2} \mathrm{Cl}_{2}\right)$ of the crude product gave pure $4.4(1 \mathrm{~g}, 78 \%)$ as a white solid: ${ }^{1} \mathrm{H}$ NMR $\left(300 \mathrm{MHz}, \mathrm{CDCl}_{3}\right)$ : $\delta 1.31-1.34(\mathrm{~d}, 2 \mathrm{H}), 1.59-1.63(\mathrm{~d}, 2 \mathrm{H}), 1.70-$ $1.74(\mathrm{~d}, 2 \mathrm{H}), 1.80-1.84(\mathrm{~d}, 2 \mathrm{H}), 2.08(\mathrm{~s}, 12 \mathrm{H}), 2.23(\mathrm{~s}, 4 \mathrm{H}), 2.30(\mathrm{~s}, 4 \mathrm{H}), 2.62(\mathrm{~s}, 4 \mathrm{H})$, 3.26-3.27 (d, $\left.18 \mathrm{H}, \mathrm{OCH}_{3}\right), 3.35-3.39$ (d, $\left.12 \mathrm{H}, \mathrm{OCH}_{2}\right), 3.77$ (s, $\left.12 \mathrm{H}, \mathrm{OOCH}_{3}\right) .{ }^{13} \mathrm{C} \mathrm{NMR}$ $\left(300 \mathrm{MHz}, \mathrm{CDCl}_{3}\right)$ : $\delta 26.53\left(\mathrm{CH}_{2}\right), 30.90\left(\mathrm{CH}_{2}\right) 34.49(\mathrm{CH}), 36.85(\mathrm{CH}), 36.08(\mathrm{CH})$, $38.92(\mathrm{CH}), 46.08(\mathrm{C}, \mathrm{cq}), 48.92(\mathrm{CH}), 49.08(\mathrm{CH}), 49.36(\mathrm{CH}), 49.50(\mathrm{CH}), 49.66(\mathrm{CH})$, $\left.51.52(\mathrm{CH})\left(\mathrm{OOCH}_{3}\right), 58.55\left(\mathrm{OCH}_{3}\right), 58.64\left(\mathrm{OCH}_{3}\right), 67.74\left(\mathrm{OCH}_{2}\right), 67.91 \mathrm{OCH}_{2}\right), 141.59$ $(\mathrm{C}=\mathrm{C}), 161.46(\mathrm{C}=\mathrm{O})$.

18-bond hexamethoxy tetraol (4.5) To a solution of $4.4(0.76 \mathrm{~g}, 0.76 \mathrm{mmol})$ in dry THF $(100 \mathrm{~mL})$ under argon was added $\mathrm{LiAlH}_{4}(1 \mathrm{~g}, 0.26 \mathrm{~mol})$ in small portions. The mixture was refluxed for $24 \mathrm{~h}$. To the cooled mixture were added $\mathrm{H}_{2} \mathrm{O}(1 \mathrm{~mL}), 15 \%$ eq. $\mathrm{NaOH}(1$ $\mathrm{mL})$ and $\mathrm{H}_{2} \mathrm{O}(3 \mathrm{~mL})$ successively with care. The mixture was then filtered through a filter aid pad while hot. The filtrate was dried over $\mathrm{Na}_{2} \mathrm{SO}_{4}$ and evaporated under reduced 
pressure to give $4.5(0.68 \mathrm{~g}, 100 \%)$ as a white solid, which was used for tosylation in next step without further purification.

20-bond hexamethoxy tetratosylate (4.6) To a cooled $\left(-5^{\circ} \mathrm{C}\right)$ solution of $4.5(0.29 \mathrm{~g}$, $0.33 \mathrm{mmol})$ in dry pyridine $(10 \mathrm{~mL})$ and $\mathrm{CH}_{2} \mathrm{Cl}_{2}(50 \mathrm{~mL})$ was slowly added $\mathrm{TsBr}(0.46 \mathrm{~g}$, $1.96 \mathrm{mmol})$. The mixture was stirred under argon atmosphere for $4 \mathrm{~h} . \mathrm{H}_{2} \mathrm{O}(20 \mathrm{~mL})$ was slowly introduced to quench the reaction. The resulting mixture was kept stirring for another $1 \mathrm{~h} . \mathrm{CH}_{2} \mathrm{Cl}_{2}(4 \times 20 \mathrm{~mL})$ was used to extract the mixture, the combined organic extracts was washed with $2 \mathrm{M} \mathrm{HCl}(4 \times 50 \mathrm{~mL})$, water $(40 \mathrm{~mL})$, saturated $\mathrm{NaHCO}_{3}$ aq. $(50 \mathrm{~mL})$, brine $(50 \mathrm{~mL})$ successively and then dried over $\mathrm{Na}_{2} \mathrm{SO}_{4}$. Evaporation of the organic solvent gave a crude product, which was chromatographed $\left(\mathrm{CH}_{2} \mathrm{Cl}_{2}\right.$ : EtOAc, $85: 15)$ to give pure $4.6(0.28 \mathrm{~g}, 57 \%)$ as a white solid: ${ }^{1} \mathrm{H}$ NMR $\left(300 \mathrm{MHz}, \mathrm{CDCl}_{3}\right): \delta$ 1.59-1.63 (d, 2H), 1.67-1.71 (d, 4H), 1.76-1.80 (m, 6H), 1.82-1.88 (m, 4H), 1.92-1.94 (d, $2 \mathrm{H}), 1.97(\mathrm{~s}, 2 \mathrm{H}), 1.99-2.00(\mathrm{~d}, 1 \mathrm{H}), 2.02-2.07$ (m, 12H), 2.15 (s, 2H), 2.19 (s, 4H), 2.22$2.29(\mathrm{~m}, 2 \mathrm{H}), 2.45\left(\mathrm{~s}, 12 \mathrm{H}, \mathrm{CH}_{3} \mathrm{CO}\right), 3.23-3.25\left(\mathrm{~m}, 18 \mathrm{H}, \mathrm{OCH}_{3}\right), 3.29-3.35(\mathrm{~m}, 12 \mathrm{H}$, $\mathrm{OCH}_{2}$ ), 3.85-3.87 (d, 4H, CH2-S), 4.01-4.14 (m, 4H, CH2-S), 7.32-7.35 (t, 8H, CH=CH), 7.73-7.76 (t, 8H, CH=CH). ${ }^{13} \mathrm{C}$ NMR $\left(300 \mathrm{MHz}, \mathrm{CDCl}_{3}\right): \delta 21.53\left(\mathrm{CH}_{3}\right), 30.72\left(\mathrm{CH}_{2}\right)$, $30.85\left(\mathrm{CH}_{2}\right), 35.54(\mathrm{CH}), 36.77(\mathrm{CH}), 37.09(\mathrm{CH}), 38.24(\mathrm{CH}), 38.55(\mathrm{CH}), 38.64(\mathrm{CH})$, $40.85(\mathrm{CH}), 48.31$ (C, cq), $48.65(\mathrm{C}, \mathrm{cq}), 48.72(\mathrm{C}, \mathrm{cq}), 48.79(\mathrm{CH}), 49.37(\mathrm{CH}), 58.59$ $(\mathrm{CH}), 58.70(\mathrm{CH}), 67.32\left(\mathrm{OCH}_{3}\right), 67.66\left(\mathrm{OCH}_{3}\right), 67.82\left(\mathrm{OCH}_{2}\right), 68.24\left(\mathrm{OCH}_{2}\right), 72.66$

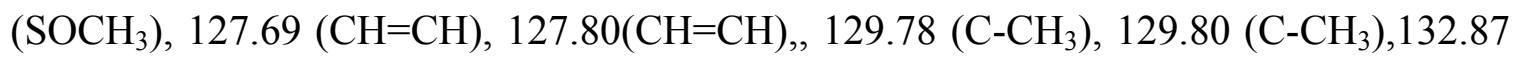
$(\mathrm{CH}=\mathrm{CH}), 133.10(\mathrm{CH}=\mathrm{CH}), 144.68(\mathrm{~S}-\mathrm{C}), 144.70(\mathrm{~S}-\mathrm{C})$.

20-bond hexamethoxy tetrathioacetate (4.7) To a solution of $4.6(0.11 \mathrm{~g}, 0.043 \mathrm{mmol})$ in dry DMSO $(5 \mathrm{~mL})$ was added $\mathrm{KSCOCH}_{3}(0.1 \mathrm{~g}, 0.86 \mathrm{mmol})$. The mixture was heated to $70{ }^{\circ} \mathrm{C}$ and kept stirring at this temperature for $24 \mathrm{~h}$ under argon. After allowing the mixture to cool down to room temperature, $\mathrm{H}_{2} \mathrm{O}(30 \mathrm{~mL})$ was slowly added and then stirred for another $1 \mathrm{~h}$. The resulting mixture was extracted with EtOAc $(8 \times 30 \mathrm{~mL})$, the combined organic extracts was dried $\left(\mathrm{Na}_{2} \mathrm{SO}_{4}\right)$ and evaporated under reduced pressure to give pure $4.7\left(0.75 \mathrm{mg}, 96 \%\right.$ ) as a pale brown solid: ${ }^{1} \mathrm{H} \mathrm{NMR}\left(300 \mathrm{MHz}, \mathrm{CDCl}_{3}\right)$ : $\delta 1.66-$ $1.70(\mathrm{~d}, 3 \mathrm{H}), 1.76-1.77$ (d, 3H), 1.79-1.81 (d, 3H), 1.84-1.88 (d, 3H), 1.91-1.95 (t, 2H), 1.96-1.98 (d, 2H), 2.01-2.06 (m, 12H), 2.13 (s, 2H), 2.17 (s, 4H), 2.22-2.27 (m, 2H), 2.30-2.31 (d, 12H), 2.79-2.88 (m, 4H, $\left.\mathrm{CH}_{2} \mathrm{~S}\right), 2.96-3.02\left(\mathrm{~m}, 2 \mathrm{H}, \mathrm{CH}_{2}-\mathrm{S}\right), 3.11-3.19$ (m, 
$\left.2 \mathrm{H}, \mathrm{CH}_{2} \mathrm{~S}\right), 3.25-3.26\left(\mathrm{~d}, 18 \mathrm{H}, \mathrm{OCH}_{3}\right), 3.32-3.39\left(\mathrm{~m}, 12 \mathrm{H}, \mathrm{OCH}_{2}\right) .{ }^{13} \mathrm{C} \mathrm{NMR}(300 \mathrm{MHz}$, $\left.\mathrm{CDCl}_{3}\right): \delta 27.73\left(\mathrm{CH}_{3} \mathrm{CO}\right), 29.57\left(\mathrm{CH}_{2}\right), 30.51\left(\mathrm{CH}_{2}\right), 30.57\left(\mathrm{CH}_{2}\right), 30.93\left(\mathrm{CH}_{2}\right), 34.89$ $(\mathrm{CH}), 35.55(\mathrm{CH}), 36.77(\mathrm{CH}), 38.89(\mathrm{CH}), 39.39(\mathrm{CH}), 41.16(\mathrm{CH}), 41.70(\mathrm{CH}), 43.64$ $(\mathrm{CH}), 48.61(\mathrm{C}, \mathrm{cq}), 48.65(\mathrm{C}, \mathrm{cq}), 48.71(\mathrm{C}, \mathrm{cq}), 48.78(\mathrm{C}, \mathrm{cq}), 49.14(\mathrm{CH}), 49.36(\mathrm{CH})$, $49.40(\mathrm{CH}), 49.49(\mathrm{CH}), 58.58(\mathrm{CH}), 58.68\left(\mathrm{OCH}_{3}\right), 58.70\left(\mathrm{OCH}_{3}\right), 67.72\left(\mathrm{OCH}_{2}\right), 67.86$ $\left(\mathrm{OCH}_{2}\right), 195.69(\mathrm{C}=\mathrm{O}), 195.90(\mathrm{C}=\mathrm{O})$.

20-bond hexamethoxy tetrathiol (4) To a solution of $4.7(68 \mathrm{mg}, 0.061 \mathrm{mmol})$ in dry THF $(10 \mathrm{~mL})$ under argon were added dry methanol $(3 \mathrm{~mL})$ and NaOMe solution $(1 \mathrm{~mL})$ (prepared by dissolving $0.05 \mathrm{~g} \mathrm{Na}$ in $1 \mathrm{~mL}$ dry methanol). The resulting mixture was kept stirring for $5 \mathrm{~m}$, after which argon-degassed $2 \mathrm{M} \mathrm{HCl}$ aqueous solution $(10 \mathrm{~mL})$ was added. The resulting mixture was extracted with $\mathrm{CH}_{2} \mathrm{Cl}_{2}(3 \times 30 \mathrm{~mL})$. The combined extracts were washed with $1 \mathrm{M}$ degassed $\mathrm{HCl}$ solution $(2 \times 50 \mathrm{~mL})$, brine $(50 \mathrm{~mL})$ and $\mathrm{H}_{2} \mathrm{O}(50 \mathrm{~mL})$ successively. Evaporation of the organic solvent after being dried over $\mathrm{NaSO}_{4}$ gave pure $4(50 \mathrm{mg}, 88 \%)$ as a white solid. ${ }^{1} \mathrm{H} \mathrm{NMR}\left(300 \mathrm{MHz}, \mathrm{CDCl}_{3}\right)$ : $\delta 1.22-$ 1.32 (m, 4H, SH), 1.70-1.73 (m, 4H), 1.77-1.86 (m, 8H), 1.95-1.98 (t, 3H), 2.01-2.08 (m, $13 \mathrm{H}), 2.12-2.25(\mathrm{~m}, 8 \mathrm{H}), 2.52-2.69\left(\mathrm{~m}, 7 \mathrm{H}, \mathrm{CH}_{2}-\mathrm{S}\right), 2.76-2.88$ (m, $\left.1 \mathrm{H}, \mathrm{CH}_{2}-\mathrm{S}\right), 3.25-3.26$ (d, $\left.18 \mathrm{H}, \mathrm{OCH}_{3}\right), 3.31-3.40\left(\mathrm{~m}, 12 \mathrm{H}, \mathrm{OCH}_{2}\right) .{ }^{13} \mathrm{C} \mathrm{NMR}\left(300 \mathrm{MHz}, \mathrm{CDCl}_{3}\right): 23.42\left(\mathrm{CH}_{2}\right.$ S), $30.73\left(\mathrm{CH}_{2}\right), 30.94\left(\mathrm{CH}_{2}\right), 31.08\left(\mathrm{CH}_{2}\right), 35.38(\mathrm{CH}), 36.78(\mathrm{CH}), 39.02(\mathrm{CH}), 43.38$ $(\mathrm{CH}), 45.57(\mathrm{CH}), 46.02(\mathrm{CH}), 48.62(\mathrm{C}, \mathrm{cq}), 48.70(\mathrm{C}, \mathrm{cq}), 48.73(\mathrm{C}, \mathrm{cq}), 48.78(\mathrm{C}, \mathrm{cq})$, $49.15(\mathrm{CH}), 49.39(\mathrm{CH}), 49.63(\mathrm{CH}), 58.61\left(\mathrm{CH}_{3} \mathrm{O}\right), 58.71\left(\mathrm{CH}_{3} \mathrm{O}\right), 67.79\left(\mathrm{CH}_{2} \mathrm{O}\right), 67.86$ $\left(\mathrm{CH}_{2} \mathrm{O}\right)$. 


\section{Reference}

(1) Smith, C. D. J. Am. Chem. Soc. 1966, 88, 4273.

(2) Mitsudo, T.; Kokuryo, K.; Shinsugi, T.; Nakagawa, Y.; Watanabe, Y.; Takegami, Y. J. Org. Chem. 1979, 44, 4492.

(3) Wooster, T. T.; Gamm, P. R.; Geiger, W. E.; Oliver, A. M.; Black, A. J.; Craig, D. C.; Paddon-Row, M. N. Langmuir 1996, 12, 6616.

(4) Black, A. J.; Wooster, T. T.; Geiger, W. E.; Paddon-Row, M. N. J. Am. Chem. Soc. 1993, 115, 7924.

(5) Jolliffe, K. A.; Langford, S. J.; Ranasinghe, M. G.; Shephard, M. J.; Paddon-Row, M. N. J. Org. Chem. 1999, 64, 1238.

(6) Oliver, A. M.; Paddon-Row, M. N. J. Chem. Soc., Perkin Trans. 1 1990, 1145.

(7) Lokan, N. R.; Paddon-Row, M. N.; Koeberg, M.; Verhoeven, J. W. J. Am. Chem. Soc. 2000, 122, 5075.

(8) Warrener, R. N. P., Ian G. Butler D. N. J. Chem. Soc., Chem. Commun. 1983, 1340.

(9) Ahmad, N.; Levison, J. J.; Robinson, S. D.; Uttley, M. F. Inorg. Synth. 1974, 15, 45 . 